\title{
Inhalt, Vol. 11, No. 3, 1988
}

\section{Contents}

Impressum 110

Obrist, R.; Battegay, M.; Obrecht, J. P.

(Basel/Schweiz)

Mediastinale Hodgkinsche Krankheit. - Eine retrospek-

tive Analyse 112

Henß, H.; Fiebig, H. H.; Schildge, J.; Arnold, H.;

Hasse, J. (Freiburg)

Phase-П-Studie der Kombination von Cisplatin und

Adriamycin bei fortgeschrittenen Pleuramesotheliomen . 118

Holtkamp, W.; Nagel, G. A. (Göttingen)

Bromokriptin beim chemotherapieresistenten, metastasierten Mammakarzinom. - Ergebnisse der AlO-Studie

GÖ-MC-BROMO 2/82 121

Hinweise für Autoren 128

Sonderbände 128

Klima, A.; Bettinger, R. (Frankfurt/M.)

Pathohistologische Serienschnittuntersuchungen bei

Patienten mit kompletter Tumorremission nach durch-

geführter zytostatischer Induktionstherapie 129

Informationen für die Klinik 138

Buchbesprechungen 140

Herrmann, G.; Hübner, K. (Frankfurt/M.)

Fibrosierende Alveolitis nach kombinierter Strahlen- und

Chemotherapie. - Ein Fallbericht 142

Kurzmitteilung

Queißer, W. (Mannheim); Herrmann, F.; Lindemann, A.

(Mainz); Anger, B. (Ulm); Hiddemann, W. (Münster);

Krey, U. (Kiel)

Therapie der Blast-Phase der chronischen granulozyti-

schen Leukämie mit Mithramycin und Hydroxykarbamid 145

Imprint 10

Obrist, R.; Battegay, M.; Obrecht, J. P. (Basle/Switzerland)

Mediastinal Hodgkin's Disease - A Retrospective Ana

lysis 112

Henß, H.; Fiebig, H. H.; Schildge, J.; Arnold, H.;

Hasse, J. (Freiburg)

Phase-II Study with the Combination of Cisplatin and 
Doxorubicin in Advanced Malignant Mesothelioma of the Pleura 118

Holtkamp, W.; Nagel, G. A. (Göttingen)

Bromocriptine with Chemotherapy Resistant, Metastatic

Breast Cancer. - Results of the AlO-Study GÖ-MC-

BROMO 2/82 121

Instructions for Authors $\quad 128$

Special Editions 128

Klima, A.; Bettinger, R. (Frankfurt/M.)

Pathohistologic Findings After Chemotherapy in Patients

with Complete Tumor Response: An Investigation on

Serial Sections 129

Clinical Information 138

Book Reviews 140

Herrmann, G.; Hübner, K. (Frankfurt/M.)

Fibrosing Alveolitis after Combined Radio- and Chemo

therapy. A Case Study 142

Short Communication

Queißer, W. (Mannheim); Herrmann, F.; Lindemann, A.

(Mainz); Anger, B. (Ulm); Hiddemann, W. (Münster);

Krey, U. (Kiel)

Therapy of the Blast Phase of Chronic Granulocytic

Leukemia with Mithramycin and Hydroxyurea 145

Bibliographischer Hinweis: Inhaltsverzeichnisse dieser Zeitschrift erscheinen regelmäßig in current contents ${ }^{\circledR}$ sowie in anderen bibliographischen Diensten. 